\title{
Controlling Electron Backstreaming Phenomena Through the Use of a Transverse Magnetic Field
}

\author{
John E. Foster* and Michael J. Patterson * \\ NASA John H. Glenn Research Center at Lewis Field. \\ Cleveland, Ohio 44135
}

\begin{tabular}{ll} 
& \multicolumn{1}{c}{ Nomenclature } \\
$B$ & $=$ magnetic field \\
$D$ & $=$ cross-field electron diffusion coefficient \\
$D_{e}$ & $=$ field-free electron diffusion coefficient \\
$E_{s}$ & $=$ electric field \\
$e$ & $=$ elementary charge of an electron \\
$J_{\text {beam }}$ & $=$ ion heam current \\
$J_{r}$ & $=$ electron cross-ficld current density \\
$J_{\text {limit }}$ & $=$ Measured ion beam current at backstreaming limit \\
$k$ & $=$ Boltzmann's constant \\
$m$ & $=$ mass of electron \\
$n_{b^{\prime}}$ & $=$ electron number density \\
$r$ & $=$ displacement normal to field line direction
\end{tabular}

Received I April 2002: revision received 12 June 2002: accepted for publication 5 August 2002 . Copyright $(\mathcal{C} 2002$ by the American Institute of Aeronautics and Astronautics. Inc. No copyright is asserted in the United States under Title 17, U.S. Code. The U.S. Government has a royalty-free license to exercise all rights under the copyright claimed herein for Governmental purposes. All other rights are reserved by the copyright owner. Copies of this paper may be made for personal or internal use, on condition that the copier pay the $\$ 10.00$ per-copy fee to the Copyright Clearance Center, Inc.. 222 Rosewood Drive, Danvers. MA 01923; include the code 0748-4658/02 $\$ 10.00$ in correspondence with the CCC.

${ }^{*}$ Research Scientist, On-Board Propulsion and Power Group. MS 301-3. 21000 Brookpark Road. Member AIAA.

${ }^{t}$ Aerospace Engineer. Member AIAA.

$$
\begin{aligned}
& T_{e}=\text { electron temperature } \\
& \left|V_{a}\right|=\text { absolute value of accelerator grid voltage } \\
& v_{c^{\prime}}=\text { electron collision frequency } \\
& \mu_{e} \quad=\text { electron mobility } \\
& \omega \quad=\text { electron cyclotron frequency }
\end{aligned}
$$

\section{Introduction}

\section{D} EEP-SPACE mission propulsion requirements can be satisfied by the use of high specific impulse systems such as ion thrusters. For such missions, however, the ion thruster will be required to provide thrust for long periods of time. To meet the long operation time and high-propellant throughput requirements, thruster lifetime must be increased. In general. potential ion thruster failure mechanisms associated with long-duration thrusting can be grouped into four areas ${ }^{1.2}$ : 1 ) ion optics failure. 2 ) discharge cathode failure 3) neutralizer failure, and 4) electron backstreaming caused by accelerator grid aperture enlargement brought on by accelerator grid crosion. The work presented here focuses on electron backstreaming. which occurs when the potential at the center of an accelerator grid aperture is insufficient to prevent the backflow of electrons into the ion thruster. The likelihood of this occurring depends on ion source operation time, plasma density, and grid voltages, as accelerator grid apertures enlarge as a result of erosion. Electrons that enter the gap between the high-voltage screen and accelerator grids are accelerated to the energies approximately equal to the beam voltage. This energetic electron beam (typically higher than $1 \mathrm{kV}$ ) can damage not only the ion source discharge cathode assembly, but also any of the discharge surfaces upstream of the ion acceleration optics that the electrons happen to impact. Indecd. past backstreaming studies have shown that near the backstreaming limit, which corresponds to the absolute value of the accelerator grid voltage below which electrons can backflow into the thruster, there is a rather sharp rise in temperature at structures such as the cathode keeper electrode (Soulas. G. C., and Rawlin. V. K., private communication. NASA Glenn Research Center, Cleveland, OII, Jeb. 2001). In this respect operation at accelerator grid voltages near the backstreaming limit is avoided.

Generally speaking, electron backstreaming is prevented by operating the accelerator grid at a sufficiently negative voltage to ensure a sufficiently negative aperture center potential. This approach can provide the necessary margin assuming an expected aperture enlargement. Operation at very negative accelerator grid voltages. however, enhances ion charge-exchange and direct impingement erosion of the accelerator grid.

The focus of the work presented here is the mitigation of electron backstreaming by the use of a magnetic tield. The presence of a magnetic field oriented perpendicular to the thruster axis can significantly decrease the magnitude of the backflowing electron current by significantly reducing the electron diffusion coefficient. Negative ion sources utilize this principle to reduce the fraction of electrons in the negative ion beam. ${ }^{3} 7$ The focus of these efforts has been on the attenuation of electron current diffusing from the discharge plasma into the negative ion extraction optics by placing the transverse magnetic field upstream of the extraction electrodes. In contrast. in the case of positive ion sources such as ion thrusters, the approach taken in the work presented here is to apply the transverse field downstream of the ion extraction system so as to prevent electrons from flowing back into the source. It was found in the work presented here that the magnetic field also reduces the absolute value of the electron backstreaming limit voltage. In this respect. the applied transverse magnetic field provides two mechanisms for electron backstreaming mitigation: 1$)$ electron current attenuation and 2) backstreaming limit voltage shift. Such a shift to less negative voltages can lead to reduced accelerator grid crosion rates.

\section{Conceptual Basis}

In general, the diffusion of electrons across a magnetic field can be described by ${ }^{8}$

$$
J_{e^{\prime}}=-e \cdot \mu_{e} \cdot n_{e^{\prime}} \cdot E_{s}-c \cdot D_{\mid} \cdot \frac{\mathrm{d} n_{e}}{\mathrm{~d} r}
$$


Under conditions where electron backstreaming occurs, the electric field opposing electron backflow is small, and thus the second term in Eq. (1) largely determines electron diffusion back into the engine. In the presence of a magnetic tield, the classical transverse diffusion coefficient can be expressed as

$$
D_{1}=\frac{D_{e}}{\left[1+\left(\omega^{2} / v_{i}^{2}\right)\right]}
$$

Here, in the limit of large magnetic field, the transverse diffusion coefficient scales as $D_{1} \propto 1 / B^{2}$. In this respect, a transverse magnetic field can significantly reduce the backflux of electrons into the thruster.

An imposed magnetic field should also introduce a shift in the electron backstreaming voltage limit. Under normal thruster conditions low-energy electrons are prevented from entering the engine as a result of repulsive center potentials at the accelerator grid. The more energetic electrons can backflow, provided they can surmount the energy barrier established by the grid potentials. Therefore, the primary electrons should be the first species to backstream because they (as compared to the thermal electrons) have the energy to cross the potential barrier when the absolute value of the accelerator grid voltage is sufficiently reduced. The imposition of a transverse magnetic field. however, can be used to shut off the backflow of this energetic component of the electron current. Because of this effect, the electron backstreaming voltage limit should occur at a less negative voltage. The reduced accelerator grid voltages need only be sufficiently negative so as to repel the thermal, low-energy electrons that can actually travel across the magnetic field lines by scattering off potential fluctuations and instabilities. ${ }^{9.10}$ The diffusion trajectories of the lower-energy electrons are perturbed more severely by thermally excited potential fuctuations $(\sim 1 \mathrm{eV})$ than the higher-energy component. This leads to a higher cross-field diffusion coefficient for the low-energy electrons.

There is a practical limit, however, as to how large the magnetic field can be for electron backstreaming mitigation. Though significant ion beam deflection can occur at very large field strength ( Tesla range), the likely issue that will place an upper limit on the magnetic field for this application is associated with the leakage of stray field lines into the discharge chamber, the likelihood of which increases with increasing applied magnetic field intensity. The leakage of field lines into the discharge chamber can disturb the ion extraction sheath at the upstream surface of the screen grid, thereby influencing the effective screen grid ion transparency.

\section{Experimental Setup}

The magnetic grid experiments took place in an aluminum vacuum tank approximately $2.2 \mathrm{~m}$ in diameter and $7.9 \mathrm{~m}$ in length. The approximate pumping speed was $110.000 \mathrm{l} / \mathrm{s}$ on xenon at $1.3 \times 10^{4} \mathrm{~Pa}$. The nominal base pressure was $1.3 \times 10^{\circ} \mathrm{Pa}$.

A $30-\mathrm{cm}$ NSTAR-type ion thruster with a modified ion optics system was used in this investigation. ${ }^{11}$ Details regarding the $30-\mathrm{cm}$ engine used in this test, the power console, and the xenon feed system can be found elsewhere. " Small-scale, 45-aperture gridlets were used in this investigation to extract a beam. As shown in Fig. 1a, the thruster's exit plane was masked down with a set of dished domes to accommodate the ion extraction gridlets. The screen gridlet consisted of a $0.762-\mathrm{mm}$-thick molybdenum plate while the accelerator grid was fabricated from 1 -mm-thick SAE 1050 steel plate. The 1050 steel was selected to increase the magnitude of the imposed downstream transverse magnetic field. The screen electrode contained 1.91 -mm-diam apertures while the accelerator grid apertures were $1.13 \mathrm{~mm}$ in diameter. The apertures were arranged in a $9 \times 5$ rectangular array that was centered about the thruster axis. The lateral center to center spacing between apertures on a given row was approximately $3.0 \mathrm{~mm}$. The aperture center to center spacing between apertures in adjacent rows was approximately $5.0 \mathrm{~mm}$. A $0.66-\mathrm{mm}$ gap between the screen and aceelerator grid was maintained using a mica washer.

The transverse magnetic field was generated by copper conductors that ran between each aperture row. The outer diameter of the

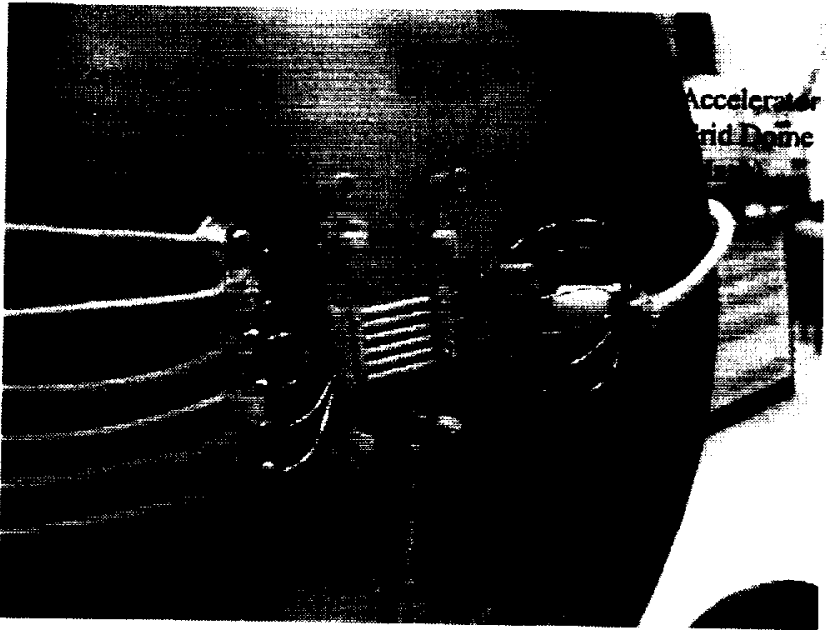

Fig. 1a Photograph of experimental setup showing masked downthruster with magnetic grid in place.

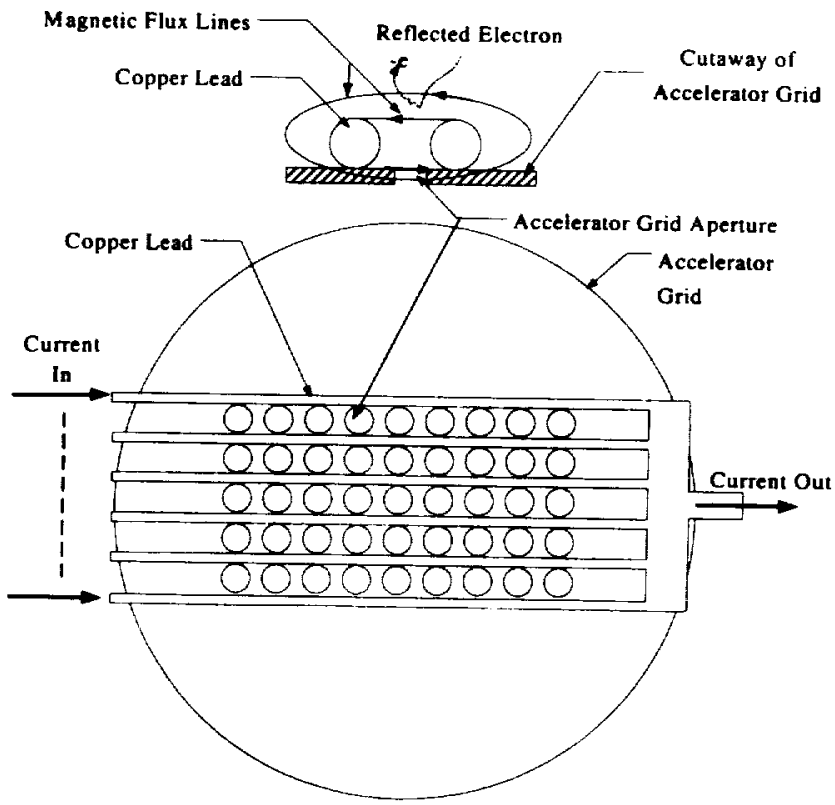

Fig. 1b Schematic depiction of the magnetic grid concept utilizing straight conductors to generate transverse magnetic field across grid apertures.

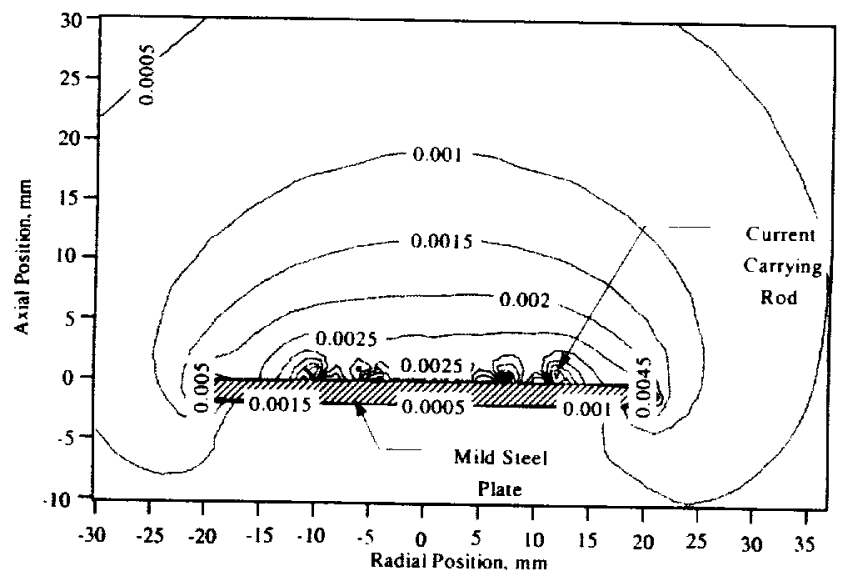

Fig. Ic Magnetic fleld contour plot generated by straight conductor configuration used in this investigation. Notice the magnetic field contours that form downstream of the gridlet. The magnetic field acts to prevent the back-diffusion of electrons into the engine. Magnetic field expressed in Teslas. 
alumina tubing that housed the copper conductors used in this investigation was $2.66 \mathrm{~mm}$. A total of six straight conductors were used, each of which was located adjacent to a row of apertures. $A$ pictorial depiction of this scheme is illustrated in Fig. 1b (also see photograph in Fig. 1a). The transverse magnetic field in a plane just above the alumina tubing that houses the copper conductors could be varied between $0 \mathrm{~T}$ and approximately $0.003 \mathrm{~T}$ as determined by Gauss-meter measurements. This magnetic field range was chosen to minimize perturbations to the ion trajectories while significantly reducing the transverse electron diffusion coefficient. Significant reductions in the transverse diffusion coefficient are possible because of the low electron-ion and electron-neutral collision frequencies downstream of the accelerator grid. As shown in the Fig. 1b, current flowing in the same direction in adjacent conductors gives rise to closed magnetic flux contours across the face of the optics. Figure 1c shows computer simulated magnetic field contours of the experimental configuration at a $12 \mathrm{~A}$ /row operating condition. As can be seen in this magnetic contour plot, the straight conductor electromagnets generate a fairly uniform, transverse magnetic field downstream of the optics. In contrast to utilizing permanent magnets, the use of the electromagnets afforded the opportunity to test the sensitivity of electron backstreaming to in situ variations in magnetic field strength.

\section{Experimental Approach}

In this work the electron backstreaming voltage limit was found by locating the "knee" of the ion beam current-accelerator grid voltage characteristic. At accelerator grid voltages less negative than at the knee, the apparent ion beam current switches from a slowly varying function of the accelerator voltage to an exponentially growing one.

\section{Results and Discussion}

It was found that as compared to the $B=0 \mathrm{~T}$ case, as the magnetic field was increased from $0 \mathrm{~T}$ to approximately $0.0030 \mathrm{~T}$, the measured ion beam current decreased between 7 and $10 \%$. The accelerator grid impingement current however did not increase, suggesting that the magnetic field was not deflecting the ions over the gap between the acceleration grids. Screen grid ion transparency measurements ${ }^{12}$ indicated a transparency reduction when the magnetic field was imposed. Screen grid ion transparency measurements determine the fraction of ion current incident at the plane of the screen grid that is actually extracted. The reductions in ion transparency are likely attributable to the leakage of magnetic field lines into the discharge plasma. This leakage of magnetic flux can affect the shape of the sheath on the upstream side of the screen grid as well as current flow into this sheath. Such a change in transparency is likely the cause of the reduction in the measured ion beam current with increasing magnetic field strength. To compensate for this effect, the discharge current was increased to bring the beam current up to its original $B=0 \mathrm{~T}$ value after each change in magnetic field intensity. The percent increase in the discharge current ranged from $17 \%$ at $0.0010^{\top} \mathrm{T}$ to $68 \%$ at approximately $0.0030 \mathrm{~T}$. Discharge voltage did not vary in response to the discharge current changes. With these changes the ion beam profile did not change from its $B=0 \mathrm{~T}$ condition. The increase in the discharge current increases the number of ions incident at the perturbed screen grid sheath so that the ion beam at the $B=0 \mathrm{~T}$ condition is recovered. In principle, the problem of field leakage into the discharge chamber can be addressed by utilizing highly permeable materials such as iron for either screen grid fabrication or as a coating for shielding purposes. Iron at $0.0020 \mathrm{~T}$ has a magnetic permeability of approximately 30 times that of cold rolled steel. ${ }^{13}$ The sensitivity of the discharge plasma near the screen grid might also be a function of ionization efficiency. Masked-down engine operation, utilized here to accommodate the small gridlets, reduced the discharge voltage and ionization efficiency (increased neutral density/pressure, increased recombination surface area) at a given discharge current.

To assess the attenuation effect of the magnetic field on electron backstreaming current, the ion beam current at the backstreaming voltage limit was measured as a function of magnetic field strength.

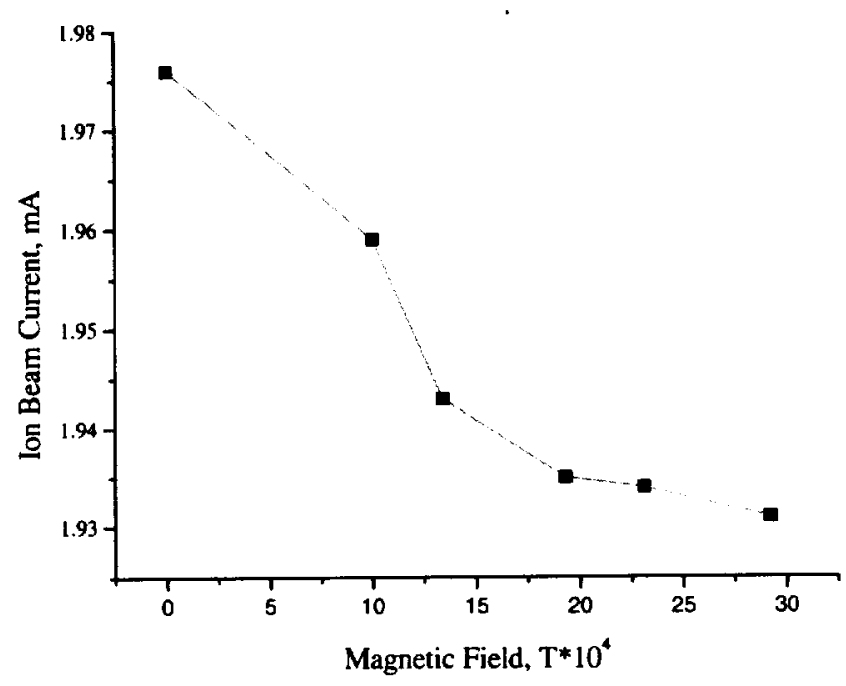

Fig. 2a Variation in the measured ion beam current at the backstreaming voltage limit as a function of magnetic field strength. Beam voltage: $1050 \mathrm{~V}$.

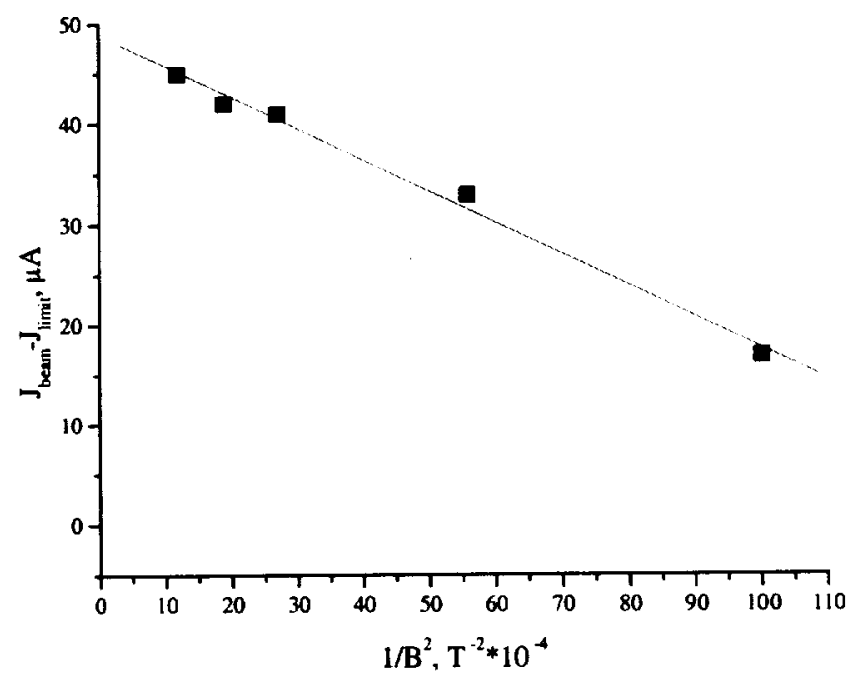

Fig. 2b Reductions in the measured ion beam current at the backstreaming limit as a function of $1 / B^{2}$. Note the variations are linear suggesting attenuation is caused by a reduction in the classical electron transverse diffusion coefficient.

Data for this study were acquired at a $1050-\mathrm{V}$ screen grid voltage and a $1.95-\mathrm{mA}$ ion beam current. The uncertainty in the ion beam current measurement was approximately $1 \mu \mathrm{A}$. The measured ion beam current at the backstreaming limit was found to decrease with increasing magnetic field strength. This monotonic decrease in the measured ion current at the backstreaming limit is illustrated in Fig. $2 \mathrm{a}$. The reduction in the ion beam current at the backstreaming limit is attributed to the attenuation of the backstreaming electron current. Figure $2 b$ illustrates the behavior of the difference between the ion beam current at $V a=-250$ (no backstreaming) and the ion beam current at the backstreaming limit as a function of $1 / B^{2}$. The linear behavior of the curve suggests that the ion current difference is caused by a reduction in electron backstreaming current brought on by a reduction in the electron transverse diffusion coefficient.

In addition to the reduction in the backstreaming current at and below of the backstreaming voltage limit, a shift in the absolute value of the electron backstreaming voltage limit to less negative values was measured. This interesting finding suggests that the absolute value of the accelerator grid voltage can be reduced to lower voltages if a transverse magnetic field is applied. thereby giving the engine additional operating margin and lifetime. Figure 3 a shows the change in the magnitude of the electron backstreaming voltage limit as a function of magnetic field strength. The data presented in this figure were taken at a beam voltage of $1050 \mathrm{~V}$ and a beam current of 


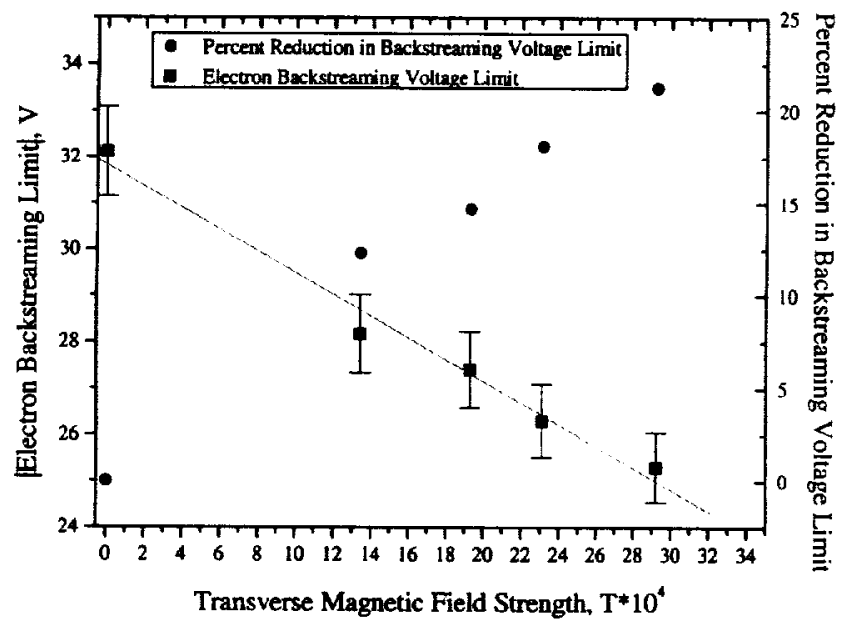

Fig. 3a Reduction in the electron backstreaming voltage limit with increasing magnetic field strength. Beam voltage $=1050 \mathrm{~V}$ and beam current $=1.95 \mathrm{~mA}$.

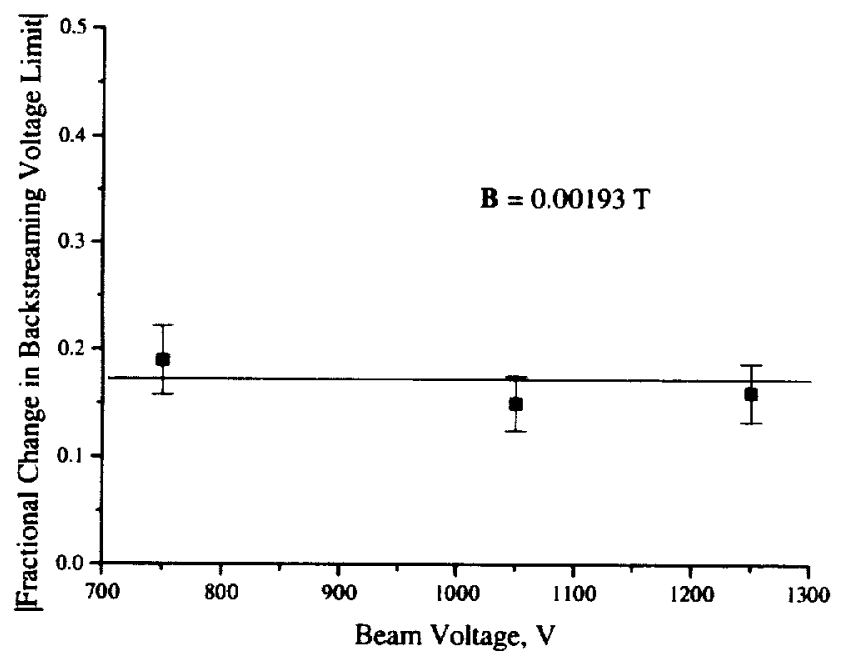

Fig. 3b Fractional change in the electron backstreaming limit as a function of beam voltage. Beam current $=1.95 \mathrm{~mA}$.

$1.95 \mathrm{~mA}$. As can be seen here, the electron backstreaming voltage limit decreases linearly with increasing transverse magnetic field strength. Also shown in this plot is the percentage reduction in the backstreaming limit with increasing magnetic field strength. As can be scen from the figure, the backstreaming voltage limit was reduced by over $20 \%$ at approximately $0.0030 \mathrm{~T}$. Using the linear fit of this data for extrapolation. reductions in the backstreaming limit of as much as $70 \%$ should be possible if a $0.01 \mathrm{~T}$ field could be imposed.

The reported reductions in the electron backstreaming voltage limit with increasing magnetic field strength can be used to add engineering margin. How do the reductions in the backstreaming voltage limit scale with the beam voltage? To access this question, a plot of the fractional change of the backstreaming limit at different beam voltages was generated as illustrated in Fig. 3b. In general. the backstreaming limit increased with increasing beam voltage. As can be seen in the figure, the percentage at which the hackstreaming voltage limit is reduced is independent of the beam voltage. These data suggest that the magnetic field reduces the electron backstreaming voltage limit by a fixed percentage rather than a fixed decrement in voltage. This percentage apparently depends on the magnitude of the magnetic field. If this scaling holds for fullscale ion optics, then, over the magnetic field range investigated in this work $(0-0.0030 \mathrm{~T})$, the backstreaming limit reduction fraction should also be approximately $20 \%$. In this regard, for example, a $154-\mathrm{V}$ backstreaming limit (NSTAR. $2.3 \mathrm{~kW}$ ) 1 might be reduced by over $31 \mathrm{~V}$ at $0.003 \mathrm{~T} \mathrm{G}$. At higher field strengths this reduction should be even higher. $\Lambda \mathrm{n}$ investigation of the implementation of this approach to a full-scale set of optics over a larger magnetic field and ion beam current density range to further validate these findings is left to a future investigation. Such an approach. however, will likely utilize a permanent magnet circuit. ${ }^{4}$

\section{Conclusions}

The effect of a transverse magnetic field. imposed on the downstream surface of the ion engine accelerator grid. on electron backstreaming was studied. Data suggest that the imposed magnetic field reduces the magnitude of the backflowing electron current at the electron backstreaming voltage limit. Additionally, the absolute value of the electron backstreaming voltage limit was found to decrease linearly with increasing magnetic tield strength. The percent reduction in the backstreaming voltage limit was independent of beam voltage. This percent reduction increased with increasing magnetic field strength. The data suggest that the transverse magnetic tield approach could be used to add engineering margin. allowing the thruster to operate at reduced accelerator grid potentials. thereby enhancing overall engine lifetime.

\section{Acknowledgments}

The authors would like to thank Robert Roman and Richard Polak for the fabrication and installation of the magnetic grid components used in this investigation. Additionally, the authors would like to thank George Soulas for maintaining stable thruster operation during these experiments.

\section{References}

'Polk. J. E.. Anderson. V. R., Brophy, J. R., Rawlin, V. K., Patterson, M. J.. Sovey, J. S., and Hamtey. J.. "An Overview of the Results from an 8200 hr. Wear Test of the NSTAR Ion Thruster," AlAA Paper 99-2446, June 1999.

${ }^{2}$ Polk, J. E.. Anderson, J. R., Brophy, J. R., Rawlin, V. K., Patterson, M. J.. Suvey, J., and Hamley, J., "An Overview of the Results from an 8200 hour Wear Test of the NSTAR Ion Thruster," AIAA Paper 99-2446, June 1999.

${ }^{3}$ Ehlers, K. W., and I eung. K. N."."Electron Suppression in a Multicusp Negative Ion Source." Applied Physics Letters, Vol. 38. No. 4, 1981, pp. 287 289.

Heung. K. N., and Ehlers, K. W., "IExraction of Volume-Produced II Ions from a Multi-Cusp Source," Review' of Scientific Instruments, Vol. 53. No. 6, 1982, pp. 803-809.

'Leung, K. N., Fhlers, K. W., and Bacal. M.. "Extraction of Volume Produced Negative Hydrogen Ions from a Multicusp Source," Review of Scientific Instruments. Vol. 54, No. 1, 1983, pp. 56-61.

${ }^{6}$ Forrester, T. A., Large lon Beams: Fundamentals of Generation and Propagation. Wiley, New York, 1988. Chap. 10, pp. 249-283.

Masanobu, T., Takeiri, Y., Asano. E.. Kaneko, O., Kuroda, T. Kawakami, H., Yamashita. Y., and Uede. T., "Production of High-Current Large-Area $\mathrm{H}^{-}$Beams by Bucket-Type Ion Source Equipped with a Magnetic Filter," IEEE Transactions on Plasma Science. Vol. 25, No. 6, 1997, pp. $1412-1417$.

${ }^{8}$ Chen. F. F., Introduction to Plasma Physics and Controlled Fusion. Plenum. New York, 1984. pp. 169-175.

${ }^{9}$ Fukumasa, O.. Naitou, H., and Sakiyama. S., "Spatial Control of Electron Energy Distribution Function in a Magnetically Filtered Multicusp Plasma Source." Japanese Journal of Applied Phrsic:s, Vol. 30, No.6A. 1991. pp. L 1063-L 1065.

${ }^{10}$ Fukumasa, O.. Naitou. H. and Sakiyama, $S$. "Control of Reactive Plasmas in a Multicusp Ion Source Equipped with a Movable Magnetic I ilter." Journal of Applied Physics, Vol. 74. No. 2. 1993, pp. 848-852.

${ }^{1}$ Sovey, J. S.. Hamley, J. A.. Haag. T. W., Patterson, M. J., Pencil. E. J., Peterson, T. T., Pinero. L. R.. Power. J. L., Rawlin, V. K., Sarmiento, C. J.. Anderson, J. R., Bond, T. A. Cardwell, G. I., and Christensen. J. A.. "Development of an Ion Thruster and Power Processor for New Millenium's Deep Space 1 Mission." AIAA Paper 97-2778, July 1997.

${ }^{12}$ Soulas, G. C., Foster, J. E., and Patterson. M. J., "Performance of Titanium Optics on a NASA $30 \mathrm{~cm}$ Ion Thruster." AIAA Paper 2000-3814, July 2000.

${ }^{13}$ Lide, D. R.. CRC Handbook of Chemistry and Physics, 72nd ed., CRC Press. Boca Raton, IL, 1991, pp. 12-96. 12-97.

${ }^{14}$ Foster, J. I., Roman, R. F., Soulas, G. C., and Patterson, M. J.. "Elec tron Backstreaming Mitigation via a Magnetic Grid." International Electric Propulsion Conference Paper 01-91, Oct. 2001. 